OPEN ACCESS

Edited by:

Giuseppe Calamita,

University of Bari Aldo Moro, Italy

Reviewed by:

Annarita Di Mise,

University of Bari Aldo Moro, Italy

Federica Cioffi,

University of Sannio, Italy

*Correspondence:

Hong Feng

18753157528@163.com

Qiang Wan

wanqiang@sdu.edu.cn

Specialty section:

This article was submitted to

Cellular Biochemistry,

a section of the journal

Frontiers in Cell and Developmental

Biology

Received: 07 December 2020

Accepted: 03 March 2021

Published: 23 April 2021

Citation:

Zhu S, Hou S, Lu Y, Sheng W

Cui Z, Dong T, Feng $H$ and Wan $Q$ (2021) USP36-Mediated

Deubiquitination of DOCK4

Contributes to the Diabetic Renal

Tubular Epithelial Cell Injury via

Wnt/ $\beta$-Catenin Signaling Pathway.

Front. Cell Dev. Biol. 9:638477.

doi: 10.3389/fcell.2021.638477

\section{USP36-Mediated Deubiquitination of DOCK4 Contributes to the Diabetic Renal Tubular Epithelial Cell Injury via Wnt/ $\beta$-Catenin Signaling Pathway}

\author{
Suwei Zhu', Shaoshuai Hou'2, Yao Lu'1, Wei Sheng ${ }^{3}$, Zhengguo Cui ${ }^{4}$, Tianyi Dong ${ }^{5}$, \\ Hong Feng ${ }^{3,6 *}$ and Qiang Wan ${ }^{7 *}$
}

${ }^{1}$ Department of Nephrology, Shandong Provincial Hospital, Cheeloo College of Medicine, Shandong University, Jinan, China, ${ }^{2}$ Department of Endocrinology, Shandong Provincial Hospital Affiliated to Shandong First Medical University, Jinan, China, ${ }^{3}$ Department of Cancer Center, Shandong Provincial Hospital, Cheeloo College of Medicine, Shandong University, Jinan, China, ${ }^{4}$ Department of Public Health, Graduate School of Medicine and Pharmaceutical Sciences, University of Toyama, Toyama, Japan, ${ }^{5}$ Department of Breast and Thyroid Surgery, Shandong Provincial Hospital Affiliated to Shandong First Medical University, Jinan, China, ${ }^{6}$ Department of Cancer Center, Shandong Provincial Hospital Affiliated to Shandong First Medical University, Jinan, China, ${ }^{7}$ Department of Endocrinology, Shandong Provincial Hospital, Cheeloo College of Medicine, Shandong University, Jinan, China

Diabetic kidney disease (DKD) has become the leading cause of end-stage renal disease but the efficacy of current treatment remains unsatisfactory. The pathogenesis of DKD needs a more in-depth research. Ubiquitin specific proteases 36 (USP36), a member of deubiquitinating enzymes family, has aroused wide concerns for its role in deubiquitinating and stabilizing target proteins. Nevertheless, the role of USP36 in diabetes has never been reported yet. Herein, we identified an increased expression of USP36 both in vitro and in vivo in diabetic renal tubular epithelial cells (TECs), and its overexpression is related to the enhanced epithelial-to-mesenchymal transition (EMT). Further investigation into the mechanisms proved that USP36 could directly bind to and mediate the deubiquitination of dedicator of cytokinesis 4 (DOCK4), a guanine nucleotide exchange factor (GEF) that could activate Wnt/ $\beta$-catenin signaling pathway and induce EMT. Our study revealed a new mechanism that USP36 participates in the pathogenesis of DKD, and provided potential intervening targets accordingly.

Keywords: ubiquitin specific proteases 36 , dedicator of cytokinesis $4, \mathrm{Wnt} / \beta$-catenin, diabetic kidney disease, deubiquitination, epithelial-to-mesenchymal transition

\section{INTRODUCTION}

Along with the worldwide prevalence of diabetes, the incidence of its major microvascular complication diabetic kidney disease (DKD) increased rapidly, and currently DKD has become the leading cause of end-stage renal disease (ESRD) (Li et al., 2017). Despite the extensive investigations and progresses were achieved in the pathogenic mechanisms of DKD during the past decades, no curative agents for DKD are clinically available yet. Current treatments only focus on the risk factors 
mediating progression of $\mathrm{DKD}$, such as hyperglycemia, hypertension and proteinuria (Nørgaard et al., 1990; de Boer et al., 2011; Mise et al., 2015). A further exploration of the pathogenesis of $\mathrm{DKD}$, and accordingly potential intervening targets, is still of significance.

Ubiquitin-proteasome system (UPS) is one of the major protein degrading mechanisms for eukaryotic cells, mainly responsible for mediating the degradation of cellular shortlived proteins, damaged or misfolded proteins (Reyes-Turcu et al., 2009). Along with the recognition of the roles USP playing in modulating cell cycle progression, gene expression, cell survival and apoptosis, UPS emerged as one crucial modulator for cellular morphological and functional homeostasis (MeyerSchwesinger, 2019). Within kidneys, UPS was also evidenced to participate in regulating ample kidney biological functions, including erythropoiesis (Meyer-Schwesinger, 2019), glucose reabsorption (Hartleben et al., 2013), salt and water balance (Wu et al., 2018). Besides, accumulating data suggested that UPS serves as an active participant in the pathogenesis of several lines of kidney pathologies, such as glomerulonephritis (Bontscho et al., 2011), acute kidney injuries (Palombella et al., 1994) and renal fibrosis (Fukasawa, 2012). However, whether UPS is also involved in the pathogenesis of DKD gained little attention, and limited studies concerning this issue reached inconsistent conclusions yet. For example, a study reported that UPS was enhanced in DKD and high glucose stimulation might promote proteasomal activities (Aghdam et al., 2013), while another report suggested that proteasomal activities were reduced in DKD (Portero-Otín et al., 1999). The concrete role of UPS in DKD still remains exploration.

Recently, ubiquitin specific proteases 36 (USP36), a member which belongs to deubiquitinating enzymes family, has aroused wide concerns for its well-identified effects in stabilizing and modifying c-Myc (Sun et al., 2015a,b). Subsequent studies revealed that USP36 is also capable of reducing Histone $\mathrm{H} 2 \mathrm{~B}$ ubiquitination at p21 locus, thereby enhancing p21 signaling and regulating cell proliferation (DeVine et al., 2018). Besides, USP36 is known to locate within mitochondria and modulate mitochondrial respiration via deubiquitinating superoxide dismutase 2 (SOD2) (Kim et al., 2011). Accompanied with the identifications of these biological functions of USP36, its role in tumorigenesis such as ovarian cancer (Li et al., 2008) and neuroblastoma (Mondal et al., 2018) has been established as well. Our previous study reported that USP36 is ubiquitously expressed within kidneys and its altered expressions participate in the pathogenesis of ischemic kidney injuries (Liu et al., 2019). Herein, we provided evidences of the increased expression of USP36 both in vitro in high glucose-induced renal tubular epithelial cells (TECs) and in vivo in human and murine DKD models, and its overexpression is related to the enhanced epithelial-to-mesenchymal transition (EMT). Further, we found that USP36 could directly bind to and mediate the deubiquitination of dedicator of cytokinesis 4 (DOCK4), a guanine nucleotide exchange factor (GEF) that could activate Wnt/ $\beta$-catenin signaling pathway and induce EMT. Our study revealed a new mechanism that USP36 participates in the pathogenesis of $\mathrm{DKD}$, and provided potential intervening targets accordingly.

\section{MATERIALS AND METHODS}

\section{Cell Culture and Treatment}

Human renal proximal tubular epithelial cells (HK-2) were obtained from the American Type Culture Collection (ATCC, Manassas, VA) and cultured in DMEM medium (Gibco, United States) supplemented with 10\% fetal bovine serum (Gibco, United States) and penicillin (100 U/ml) and streptomycin (100 mg/ml, Gibco, United States) in a humidified atmosphere of $5 \% \mathrm{CO} 2$ at $37^{\circ} \mathrm{C}$. Cells were cultured in medium containing either $5 \mathrm{mM}$ (normal glucose, NG) or $25.5 \mathrm{mM}$ (high glucose, HG) glucose for $72 \mathrm{~h}$.

\section{Human Kidney Specimens}

Renal histological sections were gained from the patients who were diagnosed as diabetic nephropathy. Human renal biopsy was done in the department of Pathology, Shandong University School of Medicine.

\section{Mouse Models}

The male C57BL/6 mice ( $8 \mathrm{w}, 21 \sim 25 \mathrm{~g}$ ) were purchased from the Shandong University Laboratory Animal Center, which were housed in controlled environments (temperature: $23 \pm 2^{\circ} \mathrm{C}$ and exposure to light from 07:00-19:00) and supplied with a standard mouse chow and water ad libitum. All animal experiments were performed according to the protocols approved by Animal Ethics Committee of Shandong University. The mice were randomly divided into the following experimental groups: normal control mice, streptozotocin-induced DKD mice. DKD groups were given an intraperitoneal injection of streptozotocin $(50 \mathrm{mg} / \mathrm{kg})$ after $12 \mathrm{~h}$ fasting for five consecutive days. Control group treated with an equal volume of citric acid buffer solution. One week after STZ injection, mice with random blood glucose levels over $16.7 \mathrm{mM}$ were considered diabetes. Four months after the diagnosis of diabetes, the mice were euthanized under anesthesia, and the kidneys were quickly removed and stored in Paraformaldehyde fixative fluid (Yu et al., 2016).

\section{SiRNAs and Plasmids}

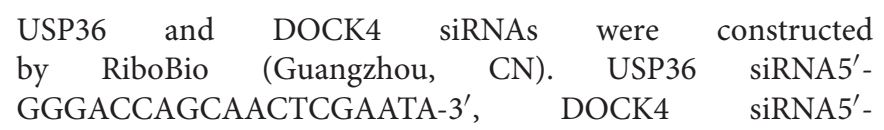
CCAGCAACGTCTTGAACCA-3'. USP36 overexpression and control plasmids were purchased from Public Protein/Plasmid Library (PPL). Ribo FECT ${ }^{\mathrm{TM}}$ CP Transfection KIT(C10511-05, RiboBio) and Lipofectamine 3000 (L3000-015, Invitrogen) were used as manufacturer's instructions described.

\section{Western Blotting}

After treatment, cells were washed with cool PBS for three times and lysed with RIPA buffer, containing protease and 
phosphatase inhibitor cocktail (P1260, Solarbio). The samples were incubated on ice for $30 \mathrm{~min}$ and then centrifuged for $30 \mathrm{~min}$ at $4^{\circ} \mathrm{C}$ at $12,000 \mathrm{rpm} / \mathrm{min}$. Collected the protein supernatant. We used $8 \%$ SDS-PAGE to divide protein in different molecular weight then the protein was transferred into $0.45 \mu \mathrm{m}$ PVDF membranes (Millipore, United States) by electroblot for $2 \mathrm{~h}$. Membranes were blocked in TBST, containing 5\% no-fat milk for $1 \mathrm{~h}$ and then incubated in primary antibodies at $4^{\circ} \mathrm{C}$ overnight. Primary antibodies were used as follows: rabbit antiUSP36 antibody (14783-1-AP, Proteintech,1:1,000), rabbit antiDOCK4 antibody (21861-1-AP, Proteintech,1:1,000), rabbit anti$\beta$-catenin antibody (51067-1-AP, Proteintech, 1:5,000), rabbit anti-E-cad antibody (20874-1-AP, Proteintech,1:5,000), rabbit anti- $\alpha$-SMA antibody (55135-1-AP, Proteintech,1:2,000). And then the membranes were incubated with the corresponding second antibody (goat anti-rabbit IgG) and detected by enhanced chemiluminescence reagents (ECL, Millipore, United States) and analyzed by Image J software.

\section{Real-Time Polymerase Chain Reaction}

Total RNA was extracted using TRIzol Reagent (Invitrogen, United States) as manufacturer's instructions described and first strand cDNA synthesis was performed using Revert Aid First Strand cDNA Synthesis Kit (Thermo scientific). Realtime polymerase chain reaction (RT-qPCR) was carried out using Ultra SYBR Mixture kit (Cwbio, CW2601M) on Roche 480II as following: firstly, $95^{\circ} \mathrm{C}$ for $2 \mathrm{~min}$ to denaturation, the second 40 cycles at $95^{\circ} \mathrm{C}$ for $15 \mathrm{~s}, 60^{\circ} \mathrm{C}$ for $1 \mathrm{~min}$, the third $95^{\circ} \mathrm{C}$ to melt and lastly $50^{\circ} \mathrm{C}$ for $30 \mathrm{~s}$ to cool down. 5'-AGCACTTTTCCCCCAGAACTG-3' (forward) and $5^{\prime}$-GGCTCCCAGATCTGCTGCTA-3' (reverse) for USP36. 5'-GAAGTGTGACGTGGACATCC-3' (forward) and $5^{\prime}$-CCGATCCACACGGAGTACTT-3' (reverse) for $\beta$ actin. $5^{\prime}$-GGGCAATGAACAACTGGGAC-3' (forward) and 5'CCTCTCCCAGGTTGGAACAC-3' (reverse) for DOCK4.

\section{Immunohistochemistry}

The sections were baked in a $68^{\circ} \mathrm{C}$ oven for $2 \mathrm{~h}$, and then dewaxed by gradient ethanol. For antigen retrieval, tissue sections were placed in sodium citrate buffer and boiled for 3 min. ORIGENE PV-9000 kit was performed according to manufacturer's instructions. And then the tissue sections were incubated with primary antibodies [rabbit anti-USP36 antibody (14783-1-AP, Proteintech, 1:200) and rabbit antiDOCK4 antibody (21861-1-AP, Proteintech, 1:100)] overnight at $4^{\circ} \mathrm{C}$. Finally, DAB staining, hematoxylin redyeing and hydrochloric acid alcohol differentiation. The images were captured with a Nikon microscope imaging system (Nikon Ti-S, Tokyo, Japan) and analyzed by Image J software.

\section{Immunofluorescence Staining}

The TECs were seeded on the chamber slides for $72 \mathrm{~h}$. Cells were washed with PBS for three times and then fixed in $4 \%$ paraformaldehyde for $20 \mathrm{~min}$ at room temperature. After washing three times with PBS, cells were permeabilized in $0.5 \%$ Triton X-100 in PBS for $20 \mathrm{~min}$ at room temperature and blocked for $1 \mathrm{~h}$ at $37^{\circ} \mathrm{C}$ by $1 \%$ goat serum (Solarbio, SL038). Cells were then incubated with rabbit anti-USP36 antibody (14783-1-AP, Proteintech,1:200) overnight at $4^{\circ} \mathrm{C}$. After washing with PBS, they were incubated with secondary antibody (Fluor488 Goat Anti-Rabbit IgG H\&L, A32731, invitrogen, 1:1,000) for $1 \mathrm{~h}$ at $37^{\circ} \mathrm{C}$. Nuclei were counterstained with DAPI for $10 \mathrm{~min}$ at room temperature. The images were captured with a Nikon microscope imaging system (Nikon Ti-S, Tokyo, Japan) and analyzed by Image J software.

\section{Immunoprecipitation Assay}

To examine ubiquitination of DOCK4, immunoprecipitation assay (IP) was carried out using a Crosslink IP kit (26147, Thermo Scientific Pierce) according to the manufacturer's instructions. In short, after treated with proteasome inhibitor MG132 (\#2194, CST,10 nM) for $6 \sim 8 \mathrm{~h}$, cells were lysed with IP Lysis Buffer. Lysates $\left(1 \mathrm{mg}\right.$ ) were incubated at $4^{\circ} \mathrm{C}$ overnight with $3 \mu \mathrm{g}$ (rabbit anti-DOCK4 antibody or rabbit control IgG antibody) and $20 \mu \mathrm{l}$ Protein A/G Agarose on a rotator. The eluent was analyzed by western blotting and analyzed by Image J software.

\section{In situ Proximity Ligation Assay}

The TECs were fixed in $4 \%$ paraformaldehyde and permeabilized in $0.75 \%$ Triton X-100. The PLA assay was carried out according to manufacturer's instructions (DUO92101, Sigma) with rabbit anti-USP36 antibody (14783-1-AP, Proteintech, 1:100), rabbit anti-ubiquitin antibody (10201-1-AP, Proteintech, 1:100) and mouse anti-DOCK4 antibody (sc-100718, santa, 1:50). The signal was visualized using an imageXpress confocal microscope (Molecular Devices) and analyzed by Image J software.

\section{Mass Spectrometry-Based Proteomics}

Differentially expressed genes with statistical significance between the two groups were identified through Volcano Plot filtering. The experiment consists of three sets of biological replicates. A total of 49 proteins were identified with a 95\% confidence in two group cells, which were cultured in medium containing either $5 \mathrm{mM}$ (normal glucose, NG) or $25.5 \mathrm{mM}$ (high glucose, HG) glucose for $72 \mathrm{~h}$. Genes were chosen for further data analysis such as clustering, GO, KEGG pathway, and protein interaction.

\section{Statement}

The brightness of all the images has been increased by $50 \%$ using photoshop software.

\section{Statistical Analysis}

Each experiment was performed at least for three times. All data were analyzed using Prism GraphPad 5.0, with Student's t-test (comparison between two groups) or one-way ANOVA (comparison among multiple groups). Error bars are represented as mean \pm S.D and statistical significance was denoted as follows: ${ }^{*} p<0.05,{ }^{* *} p<0.01,{ }^{* * *} p<0.001$, and ${ }^{* * * *} p<0.0001$. 

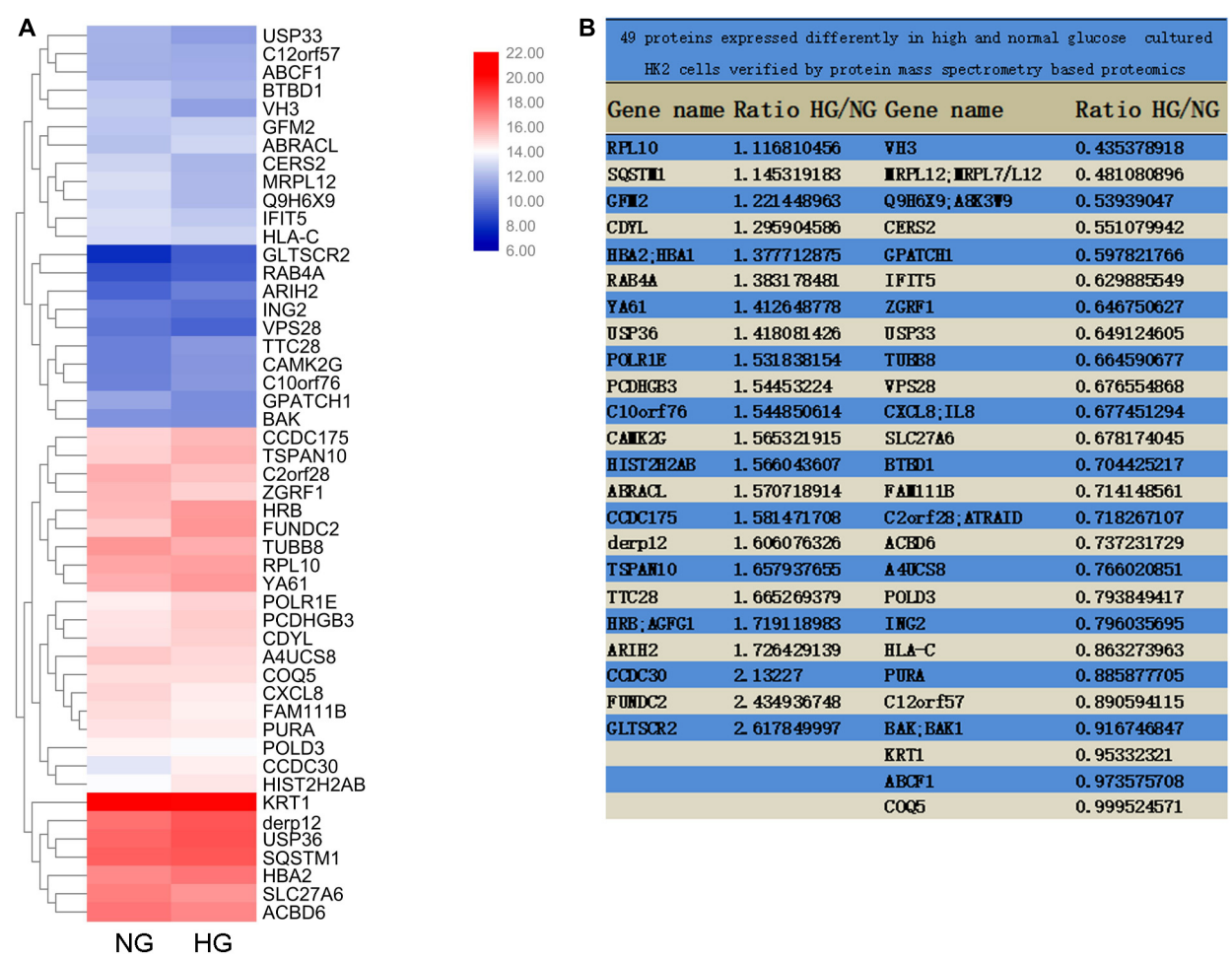

FIGURE 1 | Mass Spectrometry-Based Proteomics. (A) The heatmap of M-S analysis. Compared with the $5 \mathrm{mM}$ (normal glucose, NG) group, the expression of 49 proteins in $25.5 \mathrm{mM}$ (high glucose, HG) group was significantly different. (B) The proportion of 49 protein expression in HG and NG.

\section{RESULTS}

\section{USP36 in Renal Tubules of Diabetic Kidney Disease Patients and Mouse Models Are Significantly Increased}

To explore the pathogenic mechanism of diabetic kidney disease, we performed a mass spectrometry-based proteomics approach to search molecules that were differentially expression by high glucose in TECs. A total of 49 proteins were differentially expressed interval in high glucose-induced TECs and normal glucose controls TECs (Figures 1A,B), in which USP36 is significantly upregulated. Therefore, we were speculated that USP36 is likely to play a vital role in DKD. Immunohistochemistry staining was performed to detect the protein level of USP36 in renal tissues. Renal tissues from normal and DKD patients were used in our study. As shown in Figures 2A,B, the protein level of USP36 underwent significant elevated in human kidneys with DKD. To further explore the role of USP36 in DKD, we made a DKD mouse model by STZ treatment. The USP36 expression was also elevated in kidney tissues of STZ-induced DKD mice (Figures 2C,D). One week after STZ injection, the random blood glucose levels of mice were over $16.7 \mathrm{mM}$ (Figure 2E). The kidney metabolic parameters of STZ-induced DKD mice was demonstrated by rose urine albumin to creatinine ratio (UACR) (Figure 2F). Compared with the control mice, the renal tubules vacuolation and mesangial matrix deposition were exacerbated in STZ-induced DKD mice through Hematoxylin and eosin staining, Masson's trichrome staining and Periodic acid-Schiff staining examination (Figure 2G). These results indicated that USP36 was upregulated in DKD, which suggested that USP36 may be involved in the pathogenesis and development of DKD.

\section{USP36 in TECs Cultured in Diabetic-Like Condition Are Significantly Increased}

As shown in Figures 2H-L, RT-qPCR, western blotting and immune-fluorescence staining analysis revealed a significant increase of USP36 in TECs treated with high glucose, which indicated the possible involvement of USP36 in the progression of DKD.

\section{USP36 Participates in the Regulation of Hyperglycemia-Induced EMT of TECs}

Epithelial-to-mesenchymal transition plays a considerable role in tubulointerstitial fibrosis, which is a characteristic of diabetic kidney disease (Zhao et al., 2017). In this study, whether USP36 has an effect on EMT under high glucose condition also attracted our attention. First, the cellular USP36 contents were either reduced or elevated by transfecting USP36-silencing siRNAs or overexpressing plasmid, and the transfection efficiencies were verified by RT-qPCR and western blotting (Figures 3AC). Then, the epithelial marker E-cadherin and mesenchymal marker $\alpha$-SMA were evaluated by western blotting after gain- 

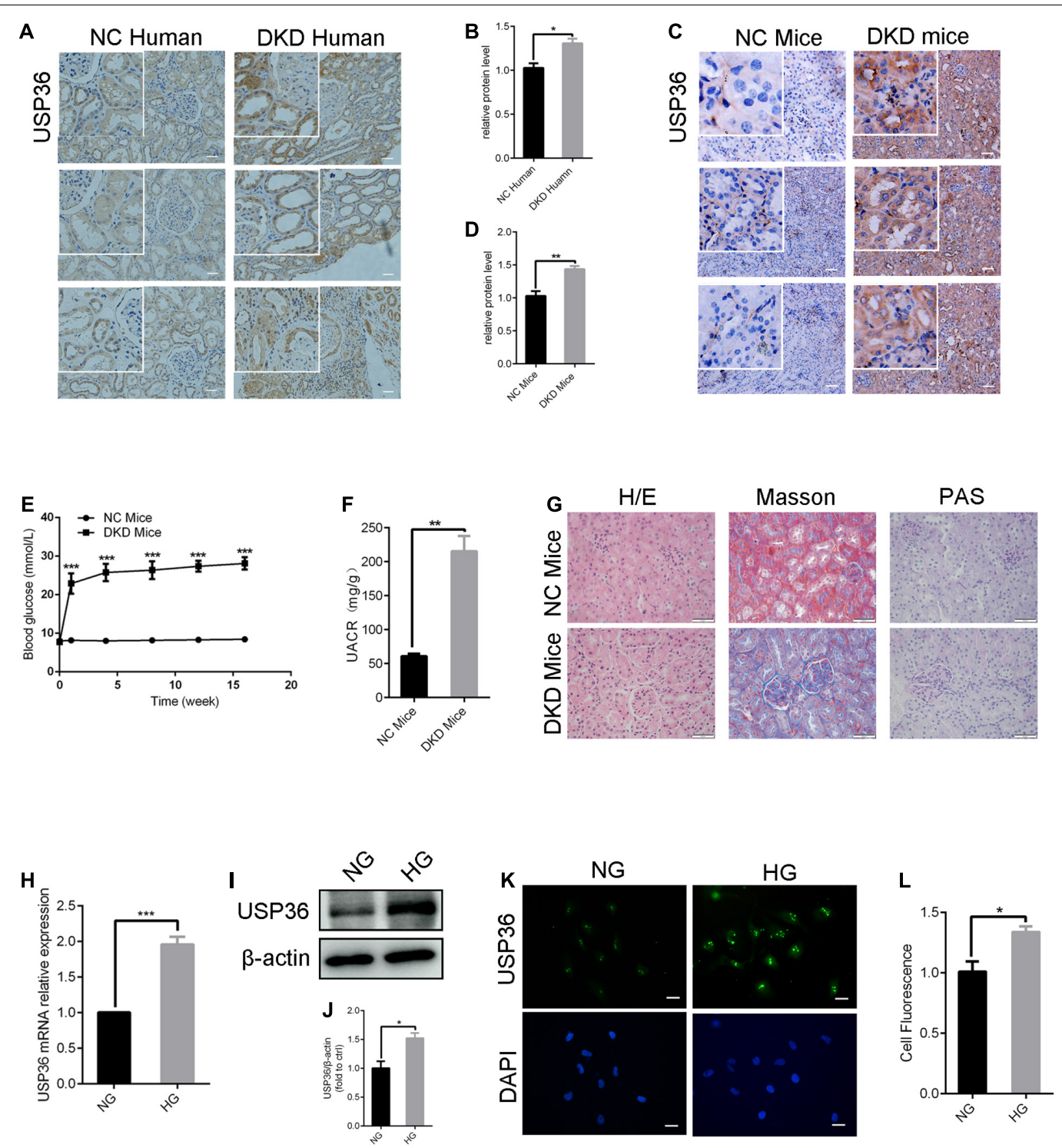

FIGURE 2 | The expression of USP36 is significantly elevated under high glucose conditions in renal TECs both in vivo and in vitro. (A,B) Immunohistochemistry staining for USP36 expression in human kidney sections. NC (normal control) represented the renal histological sections from adjacent normal tissues of patients with renal cell carcinoma. Scale bars $=100 \mu \mathrm{m}, n=3$. (C,D) Immunohistochemistry staining for USP36 expression in mouse renal tissues. Scale bars $=100 \mu \mathrm{m}$. NC (normal control) represented the renal histological sections from the normal male C57BL/6 mice, $n=3$. Scale bars, $100 \mu \mathrm{m}$. (E) The blood glucose level in NC mice and DKD mice, $n=3,{ }^{\star \star *} p<0.001$. (F) Urine albumin to creatine ratio (UACR) in STZ-induced DKD mice, $n=3,{ }^{* \star} p<0.01$. (G) Hematoxylin and eosin (H/E) staining, Masson's trichrome (Masson) staining and Periodic acid-Schiff (PAS) staining in renals from STZ-induced DKD mice. Scale bars $=50$ m, $n=3$. (H,I,K) qRT-PCR, western blotting and immunofluorescent staining were performed to detect the USP36 expression in high glucose induced TECs. Scale bars $=50 \mu \mathrm{m}$. Unpaired, two-tailed $t$ tests were used, $n=3,{ }^{\star \star \star} p<0.001$. (J,L) WB and fluorescence analysis of the protein levels of USP36 in high glucose induced TECs. Data are presented as the mean \pm S.D. $n=3,{ }^{*} p<0.05$.

or loss-of-function of USP36. As shown in Figures 3D,E, E-cadherin was significantly enhanced and $\alpha$-SMA was obviously decreased by knockdown of USP36 in TECs. By contrast, USP36 overexpressing exhibited opposite effect. Finally, E-cadherin and $\alpha$-SMA were evaluated by western blotting in TECs under high glucose. As shown in Figures 3F,G, E-cadherin was obviously decreased and $\alpha$-SMA was significantly enhanced in TECs under high glucose, while knockdown of USP36 could effectively reverse the expression of E-cadherin and $\alpha$-SMA, which suggesting that up-regulation of USP36 may be an upstream mechanism for EMT and diabetic tubulointerstitial fibrosis.

\section{USP36 Regulates the Ubiquitination Level of DOCK4}

As the regulation of EMT by USP36 was demonstrated, we continued to explore the underlying mechanisms. With this purpose, potential interactions with USP36 were speculated 

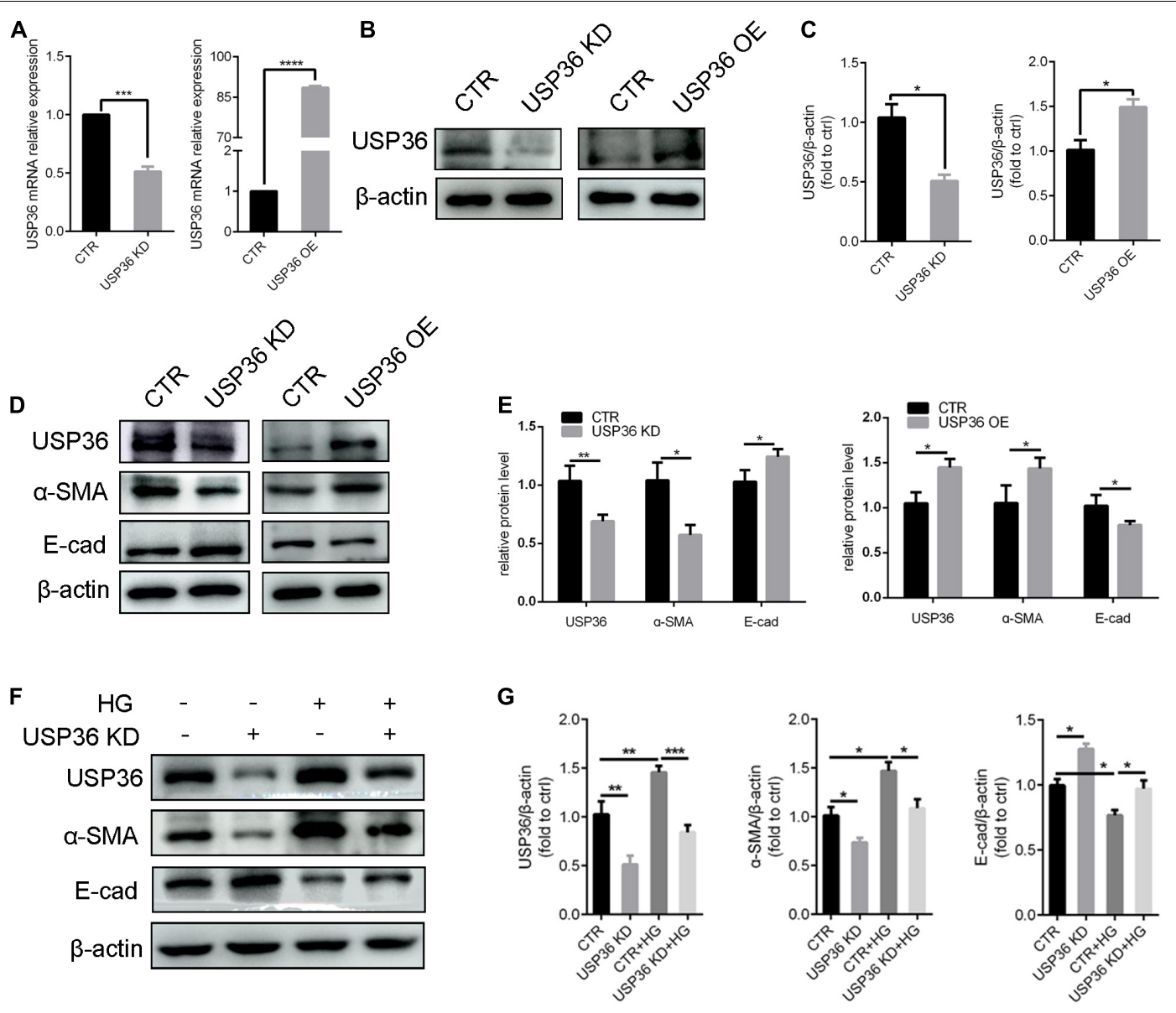

FIGURE 3 | USP36 participates in the regulation of hyperglycemia-induced EMT in TECs. (A) RT-qPCR showed knockdown or overexpression efficiency of USP36 in TECs. Unpaired, two-tailed $t$ tests were used, $n=3,{ }^{* \star \star} p<0.001,{ }^{\star \star \star \star} p<0.0001$. (B,C) WB analysis of the protein levels of USP36 after transfected with USP36-silencing siRNA or USP36-overexpressing plasmids. Data are presented as the mean \pm S.D, $n=3,{ }^{*} p<0.05$. (D,E) WB analysis of the protein levels of USP36, $\alpha$-SMA and E-cad after knockdown or overexpression of USP36. Data are presented as the mean \pm S.D. $n=3,{ }^{*} p<0.05,{ }^{* *} p<0.01$. (F,G) WB analysis of the protein levels of USP36, $\alpha$-SMA and E-cad in TECs exposed to long time high glucose following the knockdown of USP36. Data are presented as the mean \pm S.D, $n=3,{ }^{*} p<0.05,{ }^{* *} p<0.01,{ }^{* * *} p<0.001$.

in string ${ }^{1}$ (Figure 4A). Among these proteins, particularly interest is DOCK4, due to its function in activating $\mathrm{Wnt} / \beta$ catenin signaling pathway, which is associated with renal fibrosis (Xie et al., 2020). First, we evidenced that USP36 physically interacts with DOCK4 by IP and in situ proximity ligation assay (in situ PLA) (Figures 4B,C). To study the effect of USP36 on the expression of DOCK4, we evidenced the protein level of DOCK4 was significantly reduced or elevated by knockdown or overexpression of USP36 in TECs (Figures 4E,F). While the mRNA level of DOCK4 displayed no significant change (Figure 4D). Thus, we speculated that regulation of USP36 on DOCK4 expression through a post-translational mechanism. Ubiquitination is important for physiological processes which are controlled by ubiquitinating and deubiquitinating enzymes. Then, the data showed that the ubiquitination level of DOCK4 was significantly increased or decreased by knockdown or overexpression of USP36 in TECs (Figures 4G-J). Taken

${ }^{1}$ www.string-db.org together, these results indicated that USP36 could regulate the ubiquitination level of DOCK4.

\section{USP36 Regulates EMT of TECs via DOCK4-Dependent Wnt/ $\beta$-Catenin Signaling Pathway}

Previous studies demonstrated that DOCK4 is responsible for the degradation of $\beta$-catenin (Xie et al., 2020). Under this study, whether USP36 has an effect on EMT through DOCK4-dependent Wnt/ $\beta$-catenin signaling pathway in TECs under high glucose condition has aroused our attention. Consistent with USP36, immunohistochemistry staining and western blotting analysis also revealed a significant increase of DOCK4 both in vitro and in vivo under high glucose condition (Figures 5A-F). The expression of $\beta$-catenin and $\alpha$ - SMA were significantly reduced and E-cad was obviously increased when knockdown of DOCK4 or USP36 in TECs (Figures 5G-J). As shown in Figures 5K,L, western blotting 
A

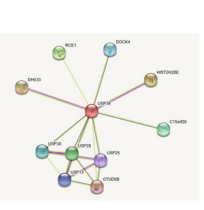

B

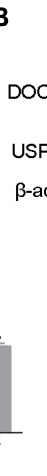

C

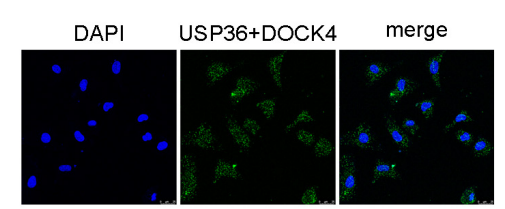

D

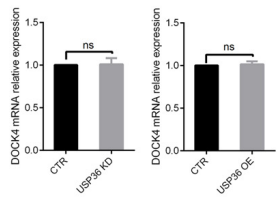

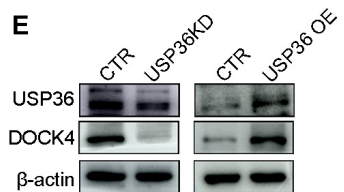
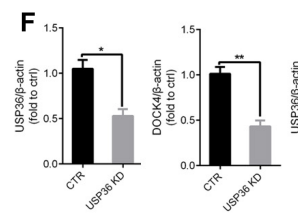

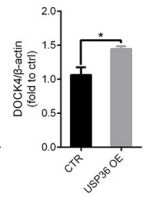

G
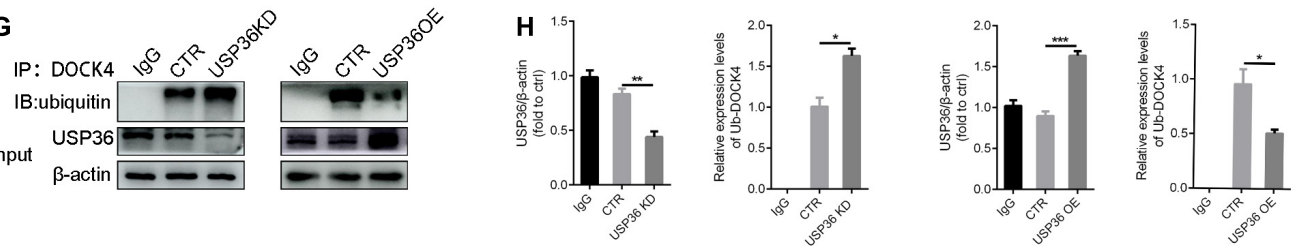

I

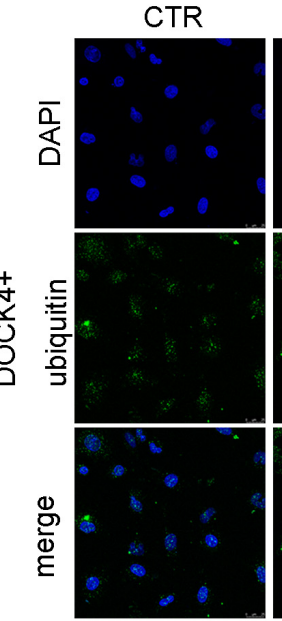

USP36 KD

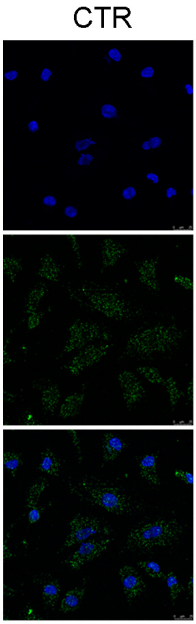

USP36OE

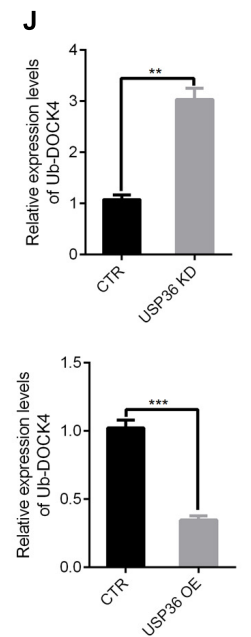

FIGURE 4 | USP36 regulates the ubiquitination level of DOCK4. (A) String website was performed to predict that USP36 can bind to DOCK4.

(B) Immunoprecipitation analysis was performed with cell lysates of TECs with anti-USP36 or anti-DOCK4 antibodies, $n=3$. (C) Interaction of USP36 and DOCK4 in TECs visualized by Duolink proximity ligation assay, $n=3$. (D) RT-qPCR analysis of the mRNA level of DOCK4 after knockdown or overexpression of USP36. Unpaired, two-tailed $t$ tests were used to compare groups, $n=3, \mathrm{~ns}, p>0.05$. (E,F) WB analysis of the protein levels of USP36 and DOCK4 in TECs after knockdown or overexpression of USP36. Data are presented as the mean \pm S.D, $n=3,{ }^{*} p<0.05,{ }^{* *} p<0.01$. (G,H) WB analysis of the protein levels of USP36 and the ub-DOCK4 in TECs after knockdown or overexpression of USP36. Data are presented as the mean \pm S.D, $n=3,{ }^{*} p<0.05,{ }^{* *} p<0.01,{ }^{* \star *} p<0.001$.

(I) Ub-DOCK4 was detected by Duolink proximity ligation assay after knockdown or overexpression of USP36. Scale bars $=25 \mu \mathrm{m}, n=3$. (J) Duolink proximity ligation assay analysis of ub-DOCK4 expression after knockdown or overexpression of USP36. Data are presented as the mean \pm S.D, $n=3$, ${ }^{\star *} p<0.01$, ${ }^{* * *} p<0.001$.

analysis revealed that the expression of $\beta$-catenin and $\alpha$ - SMA were significantly increased and E-cad was obviously reduced in high glucose-induced TECs, while these protein expressions were partial reversed by USP36 knockdown. The results revealed that DOCK4 knockdown effectively abolished USP36 overexpressioninduced EMT through suppressing Wnt/ $\beta$-catenin signaling pathway in TECs (Figures $\mathbf{5} \mathbf{M}, \mathbf{N}$ ). In brief, USP36-mediated deubiquitination of DOCK4 could contribute to the EMT in diabetic kidney disease via the $\mathrm{Wnt} / \beta$-catenin signaling pathway.

\section{DISCUSSION}

Ubiquitination and deubiquitination play a key role in maintaining cell homeostasis and are involved in the regulation of a number of biological processes. Our previous study demonstrated that as a deubiquitinating enzyme, USP36 protects TECs cells from ischemic injury by stabilizing c-Myc and SOD2 (Liu et al., 2019), implying a potential role of USP36 in renal pathology. In this study, we revealed the increased expression 

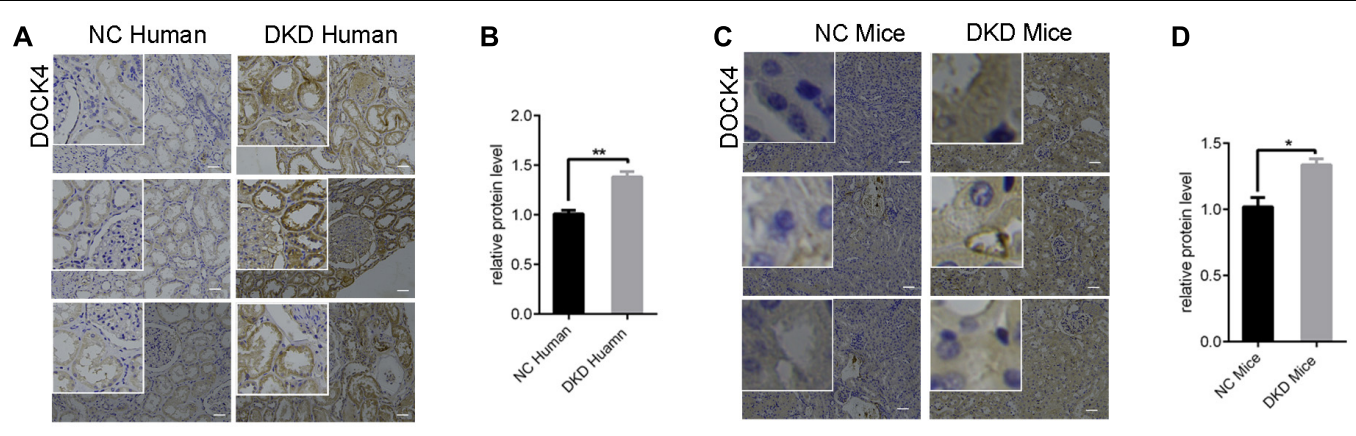
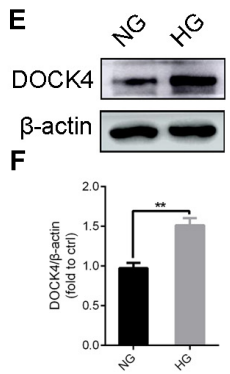
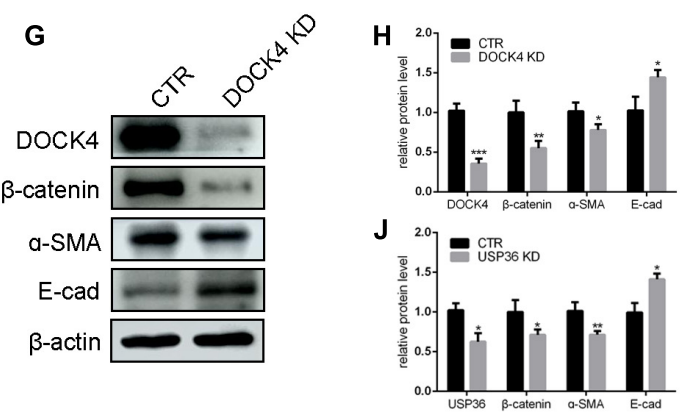

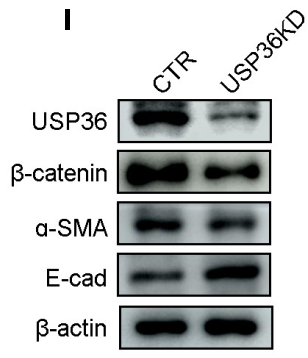

L
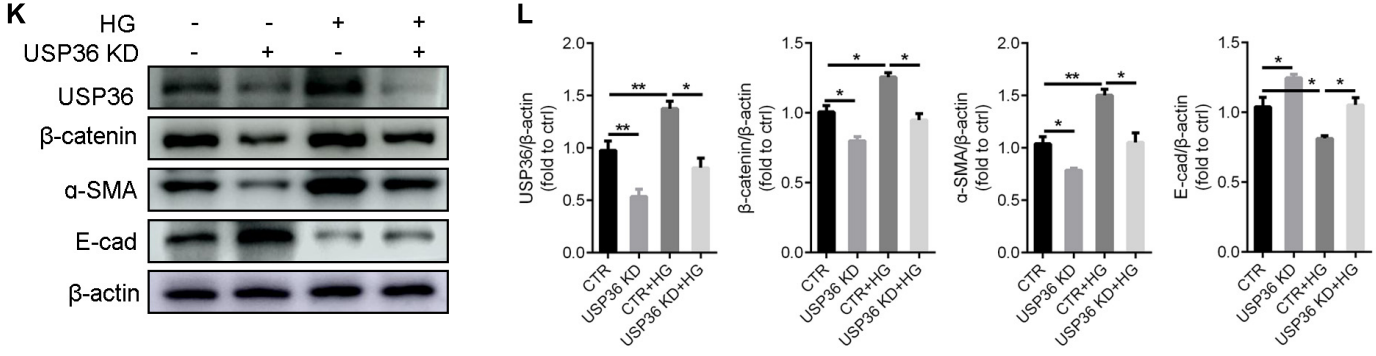

M

$\mathbf{N}$
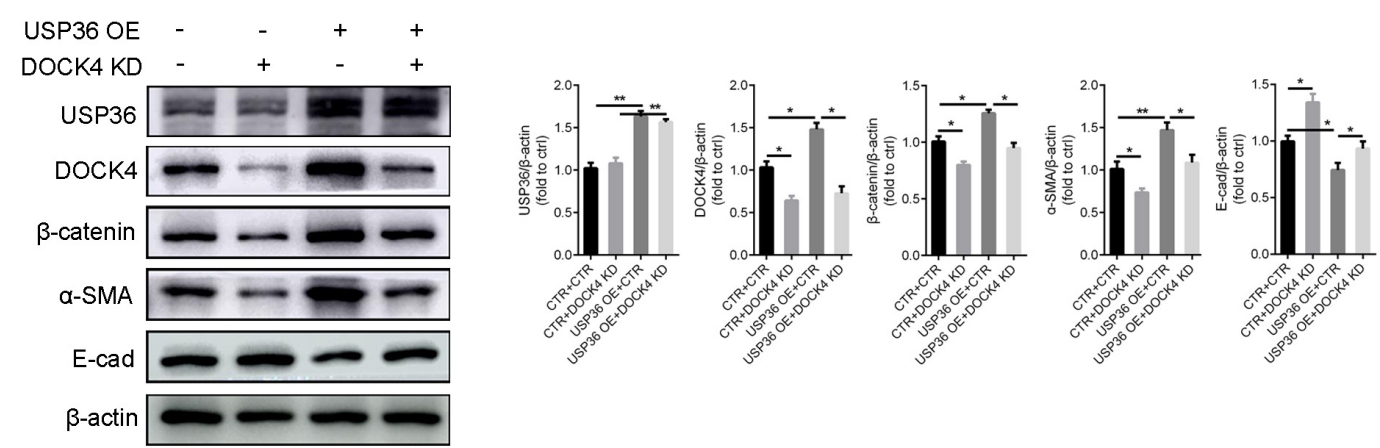

FIGURE 5 | USP36 regulates EMT induced by high glucose of TECs via DOCK4-dependent Wnt/ $\beta$-catenin signaling pathway. (A,B) Immunohistochemistry staining for DOCK4 expression in human kidney sections. Scale bars $=100 \mu \mathrm{m}, n=3$. (C,D) Immunohistochemistry staining for USP36 expression in mouse renal tissues. Scale bars $=100 \mu \mathrm{m}, n=3$. (E,F) WB analysis of the protein levels of DOCK4 in high glucose induced TECs. Data are presented as the mean \pm S.D, $n=3$, ${ }^{*} p<0.05$. (G,H) WB analysis of the protein levels of DOCK4, $\beta$-catenin, $\alpha$-SMA and E-cad in TECs after knockdown of DOCK4. Data are presented as the mean \pm S.D, $n=3$. ${ }^{*} p<0.05,{ }^{* *} p<0.01$, and ${ }^{* *} p<0.001$. (I,J) WB analysis of the protein levels of USP36, $\beta$-catenin, $\alpha$-SMA and E-cad in TECs after knockdown of USP36. Data are presented as the mean \pm S.D, $n=3$, ${ }^{*} p<0.05$, and ${ }^{* *} p<0.01$. (K,L) WB analysis of the protein levels of USP36, $\beta$-catenin, $\alpha$-SMA and E-cad in TECs exposed to long time high glucose following the knockdown of USP36. Data are presented as the mean \pm S.D, $n=3,{ }^{\star} p<0.05$, and ${ }^{\star \star} p<0.01$. (M, $\left.\mathbf{N}\right)$ WB analysis of the protein levels of USP36, DOCK4, $\beta$-catenin, $\alpha$-SMA, and E-cad after co-transfected with USP36-overexpressing and DOCK4-silencing siRNA. Data are presented as the mean \pm S.D, $n=3,{ }^{*} p<0.05$, and ${ }^{* *} p<0.01$. 
of USP36 in high glucose-induced renal tubular cell injury both in vitro and in vivo, and the role of USP36 overexpression in DKD is related to its deubiquitination effect of DOCK4, that could activate $\mathrm{Wnt} / \beta$-catenin signaling pathway and mediate EMT. Thus, these results provide evidences for a novel role of USP36 in the pathogenesis of diabetic kidney disease and identify a new target protein that is deubiquitinated by USP36.

To date, little studies concerning the ubiquitin system in the pathogenesis of DKD has been reported, moreover, these limited studies reached controversial conclusions. On the one hand, the ubiquitination activity seems to be enhanced in DKD. For example, the ubiquitin fusion protein UbA52, mainly localized in kidney tubules, was found to increase in patients with diabetes (Xie et al., 2020). Further, the expression of UBE2v1, a ubiquitin-conjugating E2 enzyme variant 1, was also increased in DKD patients and in renal tubular cells under high glucose condition, along with $\alpha$-SMA up-regulation (Pontrelli et al., 2017). In diabetic kidney, the activity of E3 ligase $\mathrm{c}-\mathrm{Cbl}$ was increased and thus resulted in podocyte injury by promoting nephrin degradation and reducing CD2AP expression (Teng et al., 2016). USP22, a deubiquitinase of USP family, was decreased in glomerular mesangial cells treated with advanced glycation end products (AGEs) (Huang et al., 2015). However, on the other hand, the enhanced process of deubiquitination has also been reported to participate in the diabetic pathology. UCH-L1, a member of the UCH protease family that deubiquitinates ubiquitin-protein conjugates, was raised in the kidneys of diabetic patients and seemed to be related to the development of DKD (Zhang et al., 2016). In STZ-induced DKD animal model, administration of proteasomal inhibitor MG132 had renal protective effect via inducing Nrf2 levels (Cui et al., 2013). Taken together, results of these studies indicate that the ubiquitination and deubiquitination are extremely complex processes in diabetes, which seems to be cell type-specific and protein-specific. Thus, we employed proteomic analysis to explore the key proteins involved in the high glucose induced renal tubular cell changes in ubiquitin system, and USP36 emerged as a candidate considering its 1.4-fold increase.

A line of proteins had been demonstrated to be targets of USP36-induced deubiquitination, including c-Myc (Sun et al., 2015b), superoxide dismutase 2(SOD2) (Kim et al., 2011), H2B (DeVine et al., 2018), PME-1 (Kim et al., 2018), DHX33 (Fraile et al., 2018), NPM, and FBL (Endo et al., 2009a). In this study, additional potential interactions of USP36 were speculated (Figure 4A). Particular interest is DOCK4, a guanine nucleotide exchange factor (GEF), on account of its essential role in $\mathrm{Wnt} / \beta$ catenin signaling (Upadhyay et al., 2008) which is associated with EMT (Xie et al., 2020). However, the way that USP36 regulates a protein expression is not limited to its direct interaction and deubiquitination modification. It has been reported that USP36 is critical for ribosomal RNA synthesis and mRNA translation through the regulation of RNA polymerase I stability (Endo et al., 2009b). In addition, USP36 has been identified as a deubiquitinase for $\mathrm{H} 2 \mathrm{Bub} 1$, which is a monoubiquitination of histone $\mathrm{H} 2 \mathrm{~B}$ enriched at actively transcribed genes and positively correlated with their expression levels (DeVine et al., 2018). Nevertheless, in this study, we demonstrated that
USP36 regulates DOCK4 expression most possibly through its deubiquitinating enzyme activity, instead of an direct regulation of transcription or translation. To elucidate the interaction between DOCK4 and USP36, immunoprecipitation and Duolink proximity ligation assay were employed (Figures 4B,C). Results showed that the DOCK4 and USP36 are more likely to have a direct connection, and ubiquitinated form of DOCK4 changed just in opposite to USP36, indicating that DOCK4 is a target of USP36 (Figures 4G,I). Furthermore, we have demonstrated for the first time that DOCK4 has a ubiquitinated form and regulated by USP36. Yet, more comprehensive and in-depth researches are needed.

DOCK4 is a member of DOCK family proteins, which are conserved across different mammalian species and emerged as a novel class of Rac/Cdc42 GTPase guanine nucleotide exchange factors (GEFs). However, members of DOCK family (termed DOCK1 to DOCK11) have been implicated to play distinct roles in diverse cell type-specific processes (Gadea and Blangy, 2014). As for DOCK4, it is a $225 \mathrm{KDa}$ protein with multiple signaling/protein-protein interaction domains (Upadhyay et al., 2008). Mutations or reduced expression of DOCK4 is related to malignancies in breast (Kobayashi et al., 2014), lung ( $Y u$ et al., 2015), brain (Debruyne et al., 2018) and blood tissues (Sundaravel et al., 2019) as well as tumor metastasis (Hiramoto et al., 2006). Dysfunctional DOCK4 is also involved in several neuropsychiatric disorders, including autism, dyslexia, mild intellectual disability (Huang M. et al., 2019), schizophrenia (Akahoshi and Yamamoto, 2018) and hearing impairment (Uehara et al., 2015). In addition, DOCK4 boosts atherosclerosis via internalization of SR-B1 and transport of LDL (Huang L. et al., 2019). However, very little is known with respect to the impact of DOCK4 in diabetes. Our present study revealed the increased DOCK4 expression both in vivo and in vitro in circumstance of high-glucose, while knockdown of DOCK4 could reverse the overexpression of USP36 induced EMT effect, indicating a role of DOCK4 played in diabetic renal fibrosis. EMT plays a considerable role in tubulointerstitial fibrosis, which is a characteristic of diabetic nephropathy. The process of EMT contains loss the expression of E-cadherin and increased the expression of $\alpha$-smooth muscle actin ( $\alpha$-SMA) (Bedi et al., 2008). Under high glucose condition, previous study reported that the expression of $\beta$-catenin was increased and have the ability to promote EMT (Guo et al., 2014). Since DOCK4 has been demonstrated to act as a scaffold protein and impedes $\beta$-catenin degradation through enhancing the release of $\beta$-catenin from degradation complex (Upadhyay et al., 2008), the regulation of EMT by DOCK4 is possibly a result of its role in modifying Wnt/ $\beta$-catenin signaling. Taken together, our have found that USP36-mediated deubiquitination of DOCK 4 contributes to the diabetic kidney disease via the Wnt/ $\beta$-catenin signaling pathway.

In summary, our results have allowed us to identify USP36 as an essential gene in diabetic renal tubular injury through, at least in part, DOCK4-dependent $\mathrm{Wnt} / \beta$-catenin signaling pathway. We describe for the first time the regulatory role of USP36 on the ubiquitination levels of DOCK4, which constitutes a new mechanism of USP36 functional implication in diabetic pathology. 


\section{DATA AVAILABILITY STATEMENT}

The original contributions presented in the study are publicly available. This data can be found here http:// proteomecentral.proteomexchange.org/cgi/GetDataset?ID=PXD 023347, accession number: PXD023347.

\section{ETHICS STATEMENT}

The studies involving human participants were reviewed and approved by the investigations were conducted in accordance with the principles of the Declaration of Helsinki and were approved by the Research Ethics Committee of Shandong University after informed consent was obtained from the patients. The patients/participants provided their written informed consent to participate in this study. The animal study was reviewed and approved by all experiments were approved by Institutional Animal Care and Use Committee of Shandong University (No. ECSBMSSDU 2018-1-045). Written informed consent was obtained from the individual(s) for the publication

\section{REFERENCES}

Aghdam, S. Y., Gurel, Z., Ghaffarieh, A., Sorenson, C. M., and Sheibani, N. (2013). High glucose and diabetes modulate cellular proteasome function: implications in the pathogenesis of diabetes complications. Biochem. Biophys. Res. Commun. 432, 339-344. doi: 10.1016/j.bbrc.2013.01.101

Akahoshi, K., and Yamamoto, T. (2018). Interstitial deletion within 7q31.1q31.3 in a woman with mild intellectual disability and schizophrenia. Neuropsychiatr. Dis. Treat. 14, 1773-1778. doi: 10.2147/NDT.S168469

Bedi, S., Vidyasagar, A., and Djamali, A. (2008). Epithelial-to-mesenchymal transition and chronic allograft tubulointerstitial fibrosis. Transplant. Rev. (Orlando). 22, 1-5. doi: 10.1016/j.trre.2007.09.004

Bontscho, J., Schreiber, A., Manz, R. A., Schneider, W., Luft, F. C., and Kettritz, R. (2011). Myeloperoxidase-specific plasma cell depletion by bortezomib protects from anti-neutrophil cytoplasmic autoantibodies-induced glomerulonephritis. J. Am. Soc. Nephrol. 22, 336-348. doi: 10.1681/ASN.2010010034

Cui, W., Li, B., Bai, Y., Miao, X., Chen, Q., Sun, W., et al. (2013). Potential role for Nrf2 activation in the therapeutic effect of MG132 on diabetic nephropathy in OVE26 diabetic mice. Am. J. Physiol. Endocrinol. Metab. 304, E87-E99. doi: 10.1152/ajpendo.00430.2012

de Boer, I. H., Sun, W., Cleary, P. A., Lachin, J. M., Molitch, M. E., Steffes, M. W., et al. (2011). Intensive diabetes therapy and glomerular filtration rate in type 1 diabetes. N. Engl. J. Med. 365, 2366-2376. doi: 10.1056/nejmoa1111732

Debruyne, D. N., Turchi, L., Burel-Vandenbos, F., Fareh, M., Almairac, F., Virolle, V., et al. (2018). DOCK4 promotes loss of proliferation in glioblastoma progenitor cells through nuclear beta-catenin accumulation and subsequent miR-302-367 cluster expression. Oncogene 37, 241-254. doi: 10.1038/onc.2017. 323

DeVine, T., Sears, R. C., and Dai, M. S. (2018). The ubiquitin-specific protease USP36 is a conserved histone $\mathrm{H} 2 \mathrm{~B}$ deubiquitinase. Biochem. Biophys. Res. Commun. 495, 2363-2368. doi: 10.1016/j.bbrc.2017.12.107

Endo, A., Kitamura, N., and Komada, M. (2009a). Nucleophosmin/B23 regulates ubiquitin dynamics in nucleoli by recruiting deubiquitylating enzyme USP36. J. Biol. Chem. 284, 27918-27923. doi: 10.1074/jbc.M109.037218

Endo, A., Matsumoto, M., Inada, T., Yamamoto, A., Nakayama, K. I., Kitamura, N., et al. (2009b). Nucleolar structure and function are regulated by the deubiquitylating enzyme USP36. J. Cell Sci. 122, 678-686. doi: 10.1242/jcs. 044461

Fraile, J. M., Campos-Iglesias, D., Rodriguez, F., Astudillo, A., Vilarrasa-Blasi, R., Verdaguer-Dot, N., et al. (2018). Loss of the deubiquitinase USP36 destabilizes of any potentially identifiable images or data included in this article.

\section{AUTHOR CONTRIBUTIONS}

SZ contributed to the design of the project, performed most of the experiments, data analysis, and prepared the manuscript. SH performed the immunohistochemistry experiments. YL performed real-time polymerase chain reaction experiments. WS and TD performed proteomics data analysis. ZC contributed to the design of the project. HF and QW helped modify the manuscript. All authors read and approved the final manuscript.

\section{FUNDING}

This study was supported by the National Natural Science Foundation of China (81770729, 91749111, and 82070756), Shandong Province Taishan Scholar Project (tsqn20161073 and ts201712092), and Shandong Province Key Research and Development Project (2016GSF201023).

the RNA helicase DHX33 and causes preimplantation lethality in mice. J. Biol. Chem. 293, 2183-2194. doi: 10.1074/jbc.M117.788430

Fukasawa, H. (2012). The role of the ubiquitin-proteasome system in kidney diseases. Clin. Exp. Nephrol. 16, 507-517. doi: 10.1007/s10157-012-0643-1

Gadea, G., and Blangy, A. (2014). Dock-family exchange factors in cell migration and disease. Eur. J. Cell Biol. 93, 466-477. doi: 10.1016/j.ejcb.2014.06.003

Guo, J., Xia, N., Yang, L., Zhou, S., Zhang, Q., Qiao, Y., et al. (2014). GSK-3beta and vitamin $\mathrm{D}$ receptor are involved in beta-catenin and snail signaling in high glucose-induced epithelial-mesenchymal transition of mouse podocytes. Cell Physiol. Biochem. 33, 1087-1096. doi: 10.1159/000358678

Hartleben, B., Widmeier, E., Suhm, M., Worthmann, K., Schell, C., Helmstadter, M., et al. (2013). aPKClambda/iota and aPKCzeta contribute to podocyte differentiation and glomerular maturation. J. Am. Soc. Nephrol. 24, 253-267. doi: 10.1681/ASN.2012060582

Hiramoto, K., Negishi, M., and Katoh, H. (2006). Dock4 is regulated by RhoG and promotes Rac-dependent cell migration. Exp. Cell Res. 312, 4205-4216. doi: 10.1016/j.yexcr.2006.09.006

Huang, K. P., Chen, C., Hao, J., Huang, J. Y., Liu, P. Q., and Huang, H. Q. (2015). AGEs-RAGE system down-regulates Sirtl through the ubiquitin-proteasome pathway to promote FN and TGF-betal expression in male rat glomerular mesangial cells. Endocrinology 156, 268-279. doi: 10.1210/en.2014-1381

Huang, L., Chambliss, K. L., Gao, X., Yuhanna, I. S., Behling-Kelly, E., Bergaya, S., et al. (2019). SR-B1 drives endothelial cell LDL transcytosis via DOCK4 to promote atherosclerosis. Nature 569, 565-569. doi: 10.1038/s41586-0191140-4

Huang, M., Liang, C., Li, S., Zhang, J., Guo, D., Zhao, B., et al. (2019). Two autism/dyslexia linked variations of DOCK4 disrupt the gene function on rac1/rap1 activation, neurite outgrowth, and synapse development. Front. Cell Neurosci. 13:577. doi: 10.3389/fncel.2019.00577

Kim, M. S., Ramakrishna, S., Lim, K. H., Kim, J. H., and Baek, K. H. (2011). Protein stability of mitochondrial superoxide dismutase SOD2 is regulated by USP36. J. Cell Biochem. 112, 498-508. doi: 10.1002/jcb.22940

Kim, S. Y., Choi, J., Lee, D. H., Park, J. H., Hwang, Y. J., and Baek, K. H. (2018). PME -1 is regulated by USP36 in ERK and Akt signaling pathways. FEBS Lett. 592, 1575-1588. doi: 10.1002/1873-3468.13039

Kobayashi, M., Harada, K., Negishi, M., and Katoh, H. (2014). Dock4 forms a complex with SH3YL1 and regulates cancer cell migration. Cell Signal. 26, 1082-1088. doi: 10.1016/j.cellsig.2014.01.027

Li, J., Olson, L. M., Zhang, Z., Bidder, M., Li, L., Nguyen, L., et al. (2008). Differential display identifies overexpression of the USP36 gene, encoding a 
deubiquitinating enzyme, in ovarian cancer. Int. J. Med. Sci. 5, 133-142. doi: 10.7150/ijms.5.133

Li, L., Wang, C., Yang, H., Liu, S., Lu, Y., Fu, P., et al. (2017). Metabolomics reveal mitochondrial and fatty acid metabolism disorders that contribute to the development of DKD in T2DM patients. Mol. Biosyst. 13, 2392-2400. doi: $10.1039 / \mathrm{c} 7 \mathrm{mb} 00167 \mathrm{c}$

Liu, Q., Sheng, W., Ma, Y., Zhen, J., Roy, S., Alvira Jafar, C., et al. (2019). USP36 protects proximal tubule cells from ischemic injury by stabilizing c-Myc and SOD2. Biochem. Biophys. Res. Commun. 513, 502-508. doi: 10.1016/j.bbrc.2019. 03.043

Meyer-Schwesinger, C. (2019). The ubiquitin-proteasome system in kidney physiology and disease. Nat. Rev. Nephrol. 15, 393-411. doi: 10.1038/s41581019-0148-1

Mise, K., Hoshino, J., Ueno, T., Hazue, R., Sumida, K., Hiramatsu, R., et al. (2015). Clinical and pathological predictors of estimated GFR decline in patients with type 2 diabetes and overt proteinuric diabetic nephropathy. Diabetes Metab. Res. Rev. 31, 572-581. doi: 10.1002/dmrr.2633

Mondal, T., Juvvuna, P. K., Kirkeby, A., Mitra, S., Kosalai, S. T., Traxler, L., et al. (2018). Sense-antisense lncRNA pair encoded by locus $6 \mathrm{p} 22.3$ determines neuroblastoma susceptibility via the USP36-CHD7-SOX9 regulatory axis. Cancer Cell 33, 417-434.e7. doi: 10.1016/j.ccell.2018.01.020 417-434.e7,

Nørgaard, K., Feldt-Rasmussen, B., Borch-Johnsen, K., Saelan, H., and Deckert, T. (1990). Prevalence of hypertension in Type 1 (insulin-dependent) diabetes mellitus. Diabetologia 33, 407-410. doi: 10.1007/bf00404089

Palombella, V. J., Rando, O. J., Goldberg, A. L., and Maniatis, T. (1994). The ubiquitin-proteasome pathway is required for processing the NF-KB1 precursor protein and the activation of NF-KB. Cell 78, 773-785. doi: 10.1016/s00928674(94)90482-0

Pontrelli, P., Conserva, F., Papale, M., Oranger, A., Barozzino, M., Vocino, G., et al. (2017). Lysine 63 ubiquitination is involved in the progression of tubular damage in diabetic nephropathy. FASEB J. 31, 308-319. doi: 10.1096/ fj.201600382RR

Portero-Otín, M., Pamplona, R., Ruiz, M. C., Cabiscol, E., Prat, J., and Bellmunt, M. J. (1999). Diabetes induces an impairment in the proteolytic activity against oxidized proteins and a heterogeneous effect in nonenzymatic protein $\mathrm{m}$ o $\mathrm{d}$ i fications in the cytosol of rat liver and kidney. Diabetes 48, 2215-2220. doi: $10.2337 /$ diabetes.48.11.2215

Reyes-Turcu, F. E., Ventii, K. H., and Wilkinson, K. D. (2009). Regulation and cellular roles of ubiquitin-specific deubiquitinating enzymes. Annu. Rev. Biochem. 78, 363-397. doi: 10.1146/annurev.biochem.78.082307.091526

Sun, X. X., He, X., Yin, L., Komada, M., Sears, R. C., and Dai, M. S. (2015a). The nucleolar ubiquitin-specific protease USP36 deubiquitinates and stabilizes c-Myc. Proc. Natl. Acad. Sci. U.S.A. 112, 3734-3739. doi: 10.1073/pnas. 1411713112

Sun, X. X., Sears, R. C., and Dai, M. S. (2015b). Deubiquitinating c-Myc: USP36 steps up in the nucleolus. Cell Cycle 14, 3786-3793. doi: 10.1080/15384101.2015. 1093713
Sundaravel, S., Kuo, W. L., Jeong, J. J., Choudhary, G. S., Gordon-Mitchell, S., Liu, H., et al. (2019). Loss of function of DOCK4 in myelodysplastic syndromes stem cells is restored by inhibitors of DOCK4 signaling networks. Clin. Cancer Res. 25, 5638-5649. doi: 10.1158/1078-0432.CCR-19-0924

Teng, B., Schroder, P., Müller-Deile, J., Schenk, H., Staggs, L., Tossidou, I., et al. (2016). CIN85 deficiency prevents nephrin endocytosis and proteinuria in diabetes. Diabetes 65, 3667-3679. doi: 10.2337/db16-0081

Uehara, D. T., Freitas, E. L., Alves, L. U., Mazzeu, J. F., Auricchio, M. T., Tabith, A. Jr., et al. (2015). A novel KCNQ4 mutation and a private IMMP2L-DOCK4 duplication segregating with nonsyndromic hearing loss in a Brazilian family. Hum. Genome Var. 2, 15038. doi: 10.1038/hgv.2015.38

Upadhyay, G., Goessling, W., North, T. E., Xavier, R., Zon, L. I., and Yajnik, V. (2008). Molecular association between beta-catenin degradation complex and Rac guanine exchange factor DOCK4 is essential for Wnt/beta-catenin signaling. Oncogene 27, 5845-5855. doi: 10.1038/onc.2008.202

Wu, Q., Moeller, H. B., Stevens, D. A., Sanchez-Hodge, R., Childers, G., Kortenoeven, M. L. A., et al. (2018). CHIP regulates aquaporin-2 quality control and body water homeostasis. J. Am. Soc. Nephrol. 29, 936-948. doi: 10.1681/ ASN.2017050526

Xie, L., Zhai, R., Chen, T., Gao, C., Xue, R., Wang, N., et al. (2020). Panax notoginseng ameliorates podocyte EMT by targeting the Wnt/beta-catenin signaling pathway in STZ-induced diabetic rats. Drug Des. Dev. Ther. 14, 527-538. doi: 10.2147/DDDT.S235491

Yu, J. R., Tai, Y., Jin, Y., Hammell, M. C., Wilkinson, J. E., Roe, J. S., et al. (2015). TGF-beta/Smad signaling through DOCK4 facilitates lung adenocarcinoma metastasis. Genes Dev. 29, 250-261. doi: 10.1101/gad.248963.114

Yu, J. W., Deng, Y. P., Han, X., Ren, G. F., Cai, J., and Jiang, G. J. (2016). Metformin improves the angiogenic functions of endothelial progenitor cells via activating AMPK/eNOS pathway in diabetic mice. Cardiovasc. Diabetol. 15:88. doi: 10.1186/s12933-016-0408-3

Zhang, H., Luo, W., Sun, Y., Qiao, Y., Zhang, L., Zhao, Z., et al. (2016). $\mathrm{Wnt} / \beta$-catenin signaling mediated-uch-11 expression in podocytes of diabetic nephropathy. Int. J. Mol. Sci. 17:1404. doi: 10.3390/ijms17091404

Zhao, Y., Yin, Z., Li, H., Fan, J., Yang, S., Chen, C., et al. (2017). MiR-30c protects diabetic nephropathy by suppressing epithelial-to-mesenchymal transition in $\mathrm{db} / \mathrm{db}$ mice. Aging Cell. 16, 387-400.

Conflict of Interest: The authors declare that the research was conducted in the absence of any commercial or financial relationships that could be construed as a potential conflict of interest.

Copyright () $2021 \mathrm{Zhu}$, Hou, Lu, Sheng, Cui, Dong, Feng and Wan. This is an open-access article distributed under the terms of the Creative Commons Attribution License (CC BY). The use, distribution or reproduction in other forums is permitted, provided the original author(s) and the copyright owner(s) are credited and that the original publication in this journal is cited, in accordance with accepted academic practice. No use, distribution or reproduction is permitted which does not comply with these terms. 